Edited by:

Alexander Borst, Max Planck Institute

of Neurobiology, Germany

Reviewed by:

Uwe Homberg, Universität Marburg, Germany

Hiromu Tanimoto, Max Planck Institute of Neurobiology, Germany

* Correspondence:

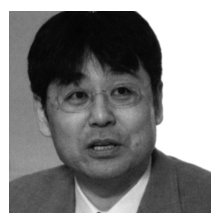

Ryohei Kanzaki is a Professor at the Research Center for Advanced Science and Technology (RCAST), The University of Tokyo, Japan. He received his Ph.D. degree in 1986, and was appointed Associate Professor at the Institute of Biological Sciences, University of Tsukuba, Japan, in 1991, following post-doctoral studies in the laboratory of John G. Hildebrand at the University of Arizona, Tucson, USA.

He was recruited as a full Professor at the Department of Mechano-Informatics,

Graduate School of Information Science and Technology, The University of Tokyo in 2004, and then moved to RCAST in 2006. His research focuses on neuroscience using insect brains as model systems. To reveal the design principles of insect brains and transfer this knowledge to engineering applications, his laboratory is set up as a multidisciplinary environment, including biology, informatics, engineering, agriculture, and chemistry. kanzaki@rcast.u-tokyo.ac.jp

\title{
Reconstruction of virtual neural circuits in an insect brain
}

\author{
Shigehiro Namiki 1,2, S. Shuichi Haupt ${ }^{1}$, Tomoki Kazawa', \\ Akira Takashima ${ }^{1}$, Hidetoshi Ikeno ${ }^{3}$ and Ryohei Kanzaki ${ }^{1 *}$ \\ 1 Research Center for Advanced Science and Technology, The University of Tokyo, Tokyo, Japan \\ 2 Graduate School of Life and Environmental Sciences, University of Tsukuba, Ibaraki, Japan \\ ${ }^{3}$ School of Human Science and Environment, University of Hyogo, Hyogo, Japan
}

The reconstruction of large-scale nervous systems represents a major scientific and engineering challenge in current neuroscience research that needs to be resolved in order to understand the emergent properties of such systems. We focus on insect nervous systems because they represent a good compromise between architectural simplicity and the ability to generate a rich behavioral repertoire. In insects, several sensory maps have been reconstructed so far. We provide an overview over this work including our reconstruction of population activity in the primary olfactory network, the antennal lobe. Our reconstruction approach, that also provides functional connectivity data, will be refined and extended to allow the building of larger scale neural circuits up to entire insect brains, from sensory input to motor output.

Keywords: digital atlas, identifiable neurons, neuron database, virtual brain

\section{INTRODUCTION}

The brain is the most important information processing and control system in more highly developed animal organisms. Because of the complexity of such systems, individual studies have normally focused on specific brain regions. For instance, we have reconstructed the neural population activity in the antennal lobe of the silkmoth in response to odorant stimuli (Namiki and Kanzaki, 2008). An important point in this work has been that it is possible to integrate the data from different experiments. This makes it possible to gradually build a database representing a neural system to some extent. However, can we reasonably hope to understand how nervous systems work without considering them as entities that can only be dissociated with some compromises? Are not the non-neural constituents of an animal's body and the environmental factors it is exposed to be included in our analyses? To work toward such an integrative approach, we have focused on insects, which have simple nervous systems compared to mammals, yet rich behavioral repertoires. Here we describe basic techniques for the reconstruction of sensory maps and future directions toward the modeling of entire behaviorally relevant neural circuits and whole insect brains.

\section{INSECT NERVOUS SYSTEM}

Experimental studies using mammalian brains are a predominant line of research in modern neuroscience. However, numerous studies using simpler invertebrate systems have contributed much to the very foundations of neuroscience (Hawkins et al., 1983; Hodgkin and Huxley, 1952; Reichardt, 1957). Some of the key reasons for using insects in brain research are:

Insect brains are convenient model systems to understand complex nervous systems in general. Although insects are unlikely to possess cognitive functions anywhere near those of mammals, they display rich behavioral repertoires comprising for example mating behavior, collision avoid- 


\section{Reconstruction}

In this review, we use the term

"reconstruction" in a broad sense for building a virtual counterpart of a real biological system. There are multiple levels for reconstruction, including cellular, local network, and whole brain levels. Different models require different experimental parameters. To build a single cell model, for example, membrane properties and the three-dimensional structure of the neuron are often required. To build a network model, the properties of synapses among neurons are required.

Identifiable neurons

Neurons or groups of neurons which exist reproducibly among different individuals of the same species. They generally have the same physiological, anatomical, and genetic properties so that we can compare and integrate the experimental data from different individuals. This feature is one of the major driving forces to focus on neural information processing in an insect brain.

Neuron database

Database for neuroscience research. Neuroscientific data are registered, shared, and analyzed systematically. The database facilitates data mining, communication among researchers and thus increases research efficiency. We create and maintain the database for neurons of silkmoth named Bombyx Neuron Database (BoND), a part of which is already available via the Internet. ance, goal-oriented behaviors, and sophisticated spatial navigation capabilities. Insects are the most successful animal group in evolution with the largest number of species. The adaptations that have evolved to many ecological niches are extremely valuable as specialized solutions and are also likely to be reflected in neural hardware. Nevertheless, the basic properties of neurons constituting insect brains are similar to those of their mammalian counterparts. Both show similar electrical properties (e.g., action potentials) and employ similar rules governing synaptic plasticity (Cassenaer and Laurent, 2007). At circuit level, extensive similarities in sensory systems have been revealed (Hildebrand and Shepherd, 1997). Thus, the investigation of these relatively simple insect brains contributes to the understanding of nervous systems in general.

Many experiments in insect nervous systems are carried out in behaving preparations. The relation between experimental treatments and behavioral outputs has traditionally placed insect neuroscience close to biologically motivated control engineering, i.e., cybernetics. The key advantage is the possibility to infer meaning though behavioral performance on a routine basis. A hypothesis based on neurophysiological results can easily be tested using behavioral experiments, simulations, and reimplementation in robotics (Delcomyn, 2004; Lindemann et al., 2008; Ritzmann and Büschges, 2007; Webb, 2002). A factor that should also always be considered is the economic importance of some insect species in sectors such as agriculture and public health.

Generally, only a limited amount of data can be obtained from a single experiment for various reasons, that are technical in nature. For instance, only a limited number of cells may be recorded simultaneously or the experimental paradigm, such as most anatomical and molecular investigations, may only allow the observation of one temporal snapshot. Under these conditions, pooling data from different individuals will be unavoidable. Whether this is reasonably possible depends critically on the amount of individual variability in the structure of interest or at least on the feasibility to exclude individual variability sufficiently from influencing the pooling process and thus the final outcome at the desired level of observation. In the mammalian neocortex, a very good correspondence between individuals can be observed in the barrel field of the primary somatosensory cortex of rodents, in which a stereotyped pattern of granular zones (barrels), each of them a single cortical column, represent defined whiskers on the snout. Using this map, physiological and morphological data of single neurons can be merged across individuals (Bjaalie et al., 2005). Other cases, in which a close correspondence between individuals can even be carried further are the decapod stomatogastric nervous system and the nervous system of Caenorhabditis elegans. In both, the number of neurons and their synaptic connectivity are nearly constant across individuals (Marder and Bucher, 2007; White et al., 1986).

From these two examples, the importance of identifiable neurons in invertebrate systems is evident. Identifiable neurons are cells that can be uniquely recognized in each individual of a species, allowing the accumulation of physiological, morphological, pharmacological, and genetic information from different experiments. The concept of identifiable neurons has given a strong impetus to the research in insect brains in the past (Comer and Robertson, 2001) and gives such preparations an advantage over vertebrate systems, although some identifiable neurons are also known in fish, notably the pair of Mauthner cells that are command neurons involved in escape behavior (Eaton et al., 2001).

\section{RECONSTRUCTION OF INSECT SENSORY NETWORK}

Due to the availability of identifiable neurons and identifiable structures and its small size, the analysis based on compilation of the physiological and morphological data has been common in a traditional insect brain research. The success probability of in vivo intracellular recording is low and experimenters are not always able to record from the neurons of interests. If all recorded neurons are registered on the database, a comprehensive analysis is possible by analyzing the data obtained from different experiments performed by different experimenters. This procedure can be facilitated by data compilation, administration, and visualization operated on the same platform, utilizing neuroinformatics. For example, we have developed a neuron database for male silkmoth brain and approximately 1,200 physiological and/or morphological data have been registered over the last 10 years (Kazawa et al., 2008). The amount of the contents is the largest among databases which focus on a single animal species. The compiled data registered by 18 different experimenters have achieved several important results. For example, 153 antennal lobe local interneurons have been registered in the database and classified into five types (Seki and Kanzaki, 2008). This classification did correspond to the computational classification by fractal dimension calculated using the box counting method (Urata et al., 2006). Anatomical identification and classification have also been 


\section{Digital atlas}

An anatomical map in a vector format. The similarity of the nervous systems among different individuals turns such an atlas into an invaluable tool facilitating data comparison and integration. done in neuronal populations in the mushroom body, the center of learning and memory, and the lateral accessory lobe, the premotor network in the insect brain, based on 109 and 120 registered neurons, respectively (Fukushima and Kanzaki, 2009). These studies made use of the data obtained by different experimenters.

In previous work, we have collected single-cell recording data obtained from different experiments under the same experimental conditions (Namiki and Kanzaki, 2008). We have obtained recordings from 38 identified projection neurons and covered 32 of the total number of $60 \pm 2$ glomeruli. We have subsequently merged these data with reference to an anatomical map (Kazawa et al., 2009) and reconstructed stimulusinduced population activity. The reconstruction approach has been utilized to analyze several sensory representations in insects and is particularly suitable when the structure of interest has clear anatomical landmarks. For example, the antennal lobe and the central body are composed of anatomically defined subunits facilitating reconstruction (Heinze and Homberg, 2007; Namiki and Kanzaki, 2008). In contrast, the reconstruction of seemingly relatively unstructured neuropils such as the lateral protocerebrum requires more involved image processing techniques (Jefferis et al., 2007; Tanaka et al., 2004; Troyer et al., 1994). For example, in the study of a mechanosensory representation in the terminal abdominal ganglion of crickets, the neuropil structures of interest are not defined by a clear anatomical delineation and maps have to be generated based on landmarks not related to the structure itself. This type of integration process for experimental data from different individuals to reconstruct a sensory map has first been applied to the cricket cercal system. Crickets have a pair of appendages on the abdomen, the cerci, which bear sets of filiform hairs with different preferences for wind direction and frequency. Primary sensory neurons of these hairs send axonal projections into the terminal abdominal ganglion. Notably, sensory neurons with similar response profiles have similar axonal projection patterns in the ganglion (Bacon and Murphey, 1984). The group digitized the neuronal morphology and pooled the data following a linear transformation with reference to the outer surface of each ganglion. Using these methods, it was possible to reveal the detailed topographic architecture of the system in which different types of information are processed in distinct subregions of the projection area of the filiform hairs' mechanoreceptors (Jacobs and Theunissen, 1996, 2000; Troyer et al., 1994). This case study has proven that landmarks not belonging to the structure under investigation can be employed to map data into a common geometry for pooling and reconstruction of a sensory representation.

The antennal lobe, the first order olfactory processing center in insects, contains clearly anatomically segregated structures, called glomeruli. Each primary sensory neuron on the antennae expresses one of approximately 60 types of olfactory receptor proteins in the silkmoth. Notably, sensory neurons which express the same types of receptor proteins send axonal projections to the same glomeruli and make synapses with central neurons. As such, glomeruli are the functional units of olfactory processing (Vosshall and Stocker, 2007). Individual glomeruli have characteristic size, shape, and relative positions and are identifiable across different individuals (Rospars, 1983). Because of the constancy of glomerular organization, a digital atlas of the antennal lobe can be made (Galizia et al., 1999a; Kazawa et al., 2009). Utilizing such anatomical maps, for example, response profiles of sensory neurons have been reconstructed on the digital atlas in the fly (Hallem and Carlson, 2006).

We have performed a reconstruction of odorevoked dynamics among projection neurons (Namiki and Kanzaki, 2008), which are the second order neurons in the insect olfactory system and functional equivalents of mitral/tufted cells in the mammalian brain. At first, we have examined the morphological characteristics of this neuronal population and revealed that most of them innervated to only a single glomerulus. This indicates that by sampling this neuronal population, we are able to measure the output information of the antennal lobe with single glomerular resolution. Projection neurons innervating the same glomeruli show similar olfactory response profiles as shown in other insect species (Reisenman et al., 2005; Wilson et al., 2004). Due to this observation, the reconstruction approach, i.e., integrating data from different individuals to assemble a virtual neural circuit, is feasible in the moth antennal lobe. Utilizing the digital atlas of this system (Kazawa et al., 2009), we identified the glomeruli innervated by stained projection neurons. By mapping the dynamics of firing rate onto the glomeruli, we reconstructed the olfactory representation based on a population of projection neurons (Figure 1). In the reconstructed map, odor-evoked firing rate changes are represented as color in each glomerulus. Temporal aspects of network dynamics in the virtual projection neuron population can be analyzed. For example, this population may discriminate given olfactory stimulations at each time (Figure 2). From the reconstructed map, it 


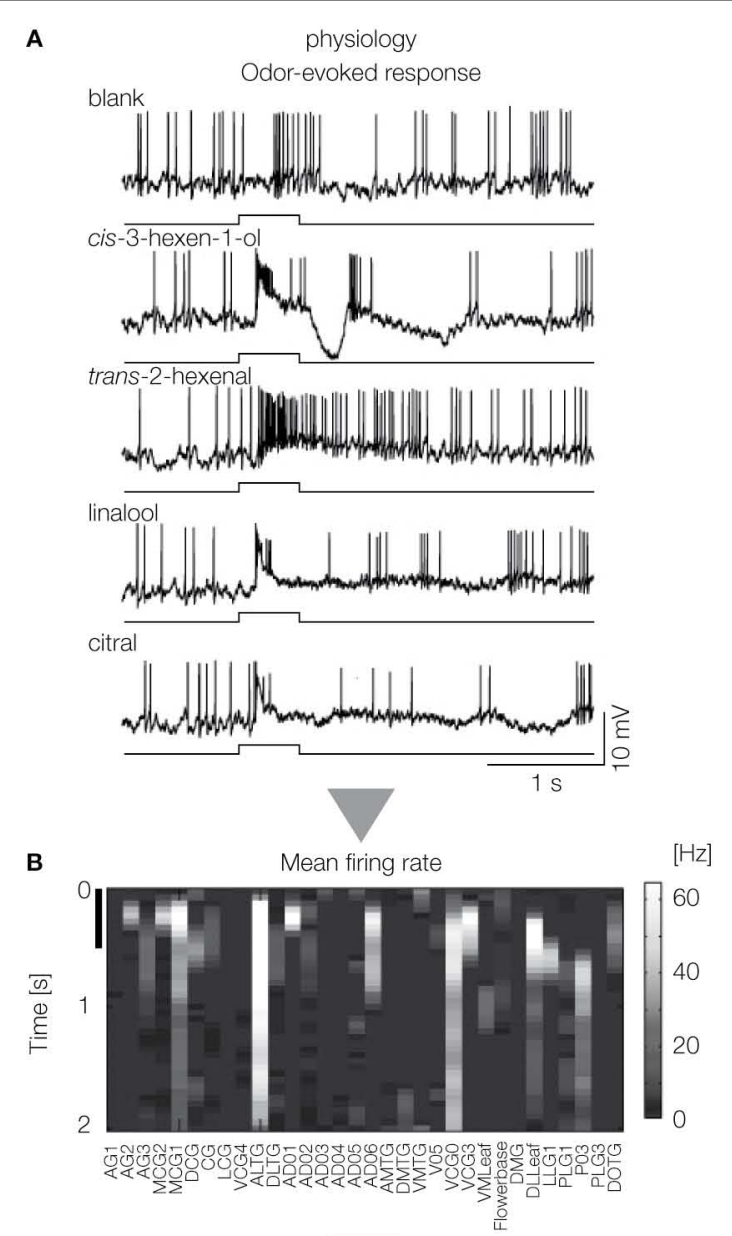

C

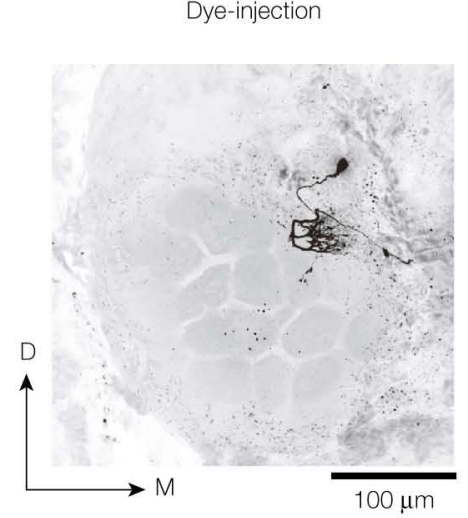

D Identification of glomeruli

$E$

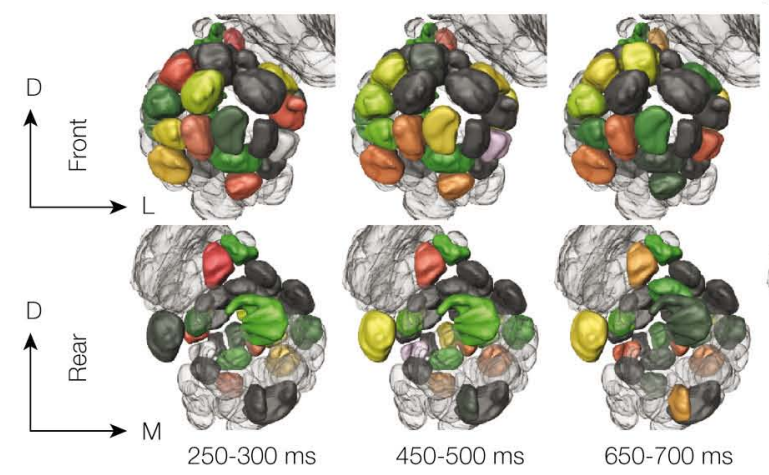

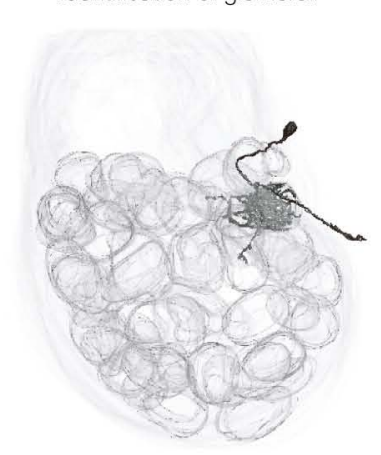

$\sqrt{ }$
Reconstruction of network dynamics

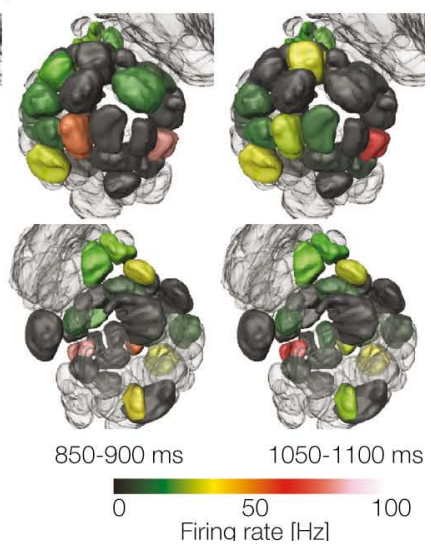

Figure 1 | Reconstruction of the population activity of the antennal lobe projection neurons. Data were obtained by in vivo intracellular recording and staining with glass microelectrodes. Odor-evoked firing activity is monitored (A), averaged across trials, and mean firing rate for each time window is calculated (B). Row and column mean time and glomeruli innervated by the stained projection neurons, respectively. Colors in the figure represent mean firing rate change for each projection neuron in response to cis-3-hexen-1-ol. Scale on the vertical axis represents the time period of odor presentation $(500 \mathrm{~ms})$. Dye-filled neurons are imaged by confocal laser scanning microscopy (C), and the glomeruli innervated by the stained neurons are identified (D). Pooled data are integrated with geometrical information using a digital atlas (E). Reconstructed odor-evoked activity by a virtual projection neuron population in response to cis-3-hexan-1-ol is shown. Upper and lower images show anterior and posterior views of the reconstructed dynamics. The number at the bottom is the time elapsed after the onset of odor presentation. Five time points are shown. $D$, dorsal; $L$, lateral; $M$, medial. 


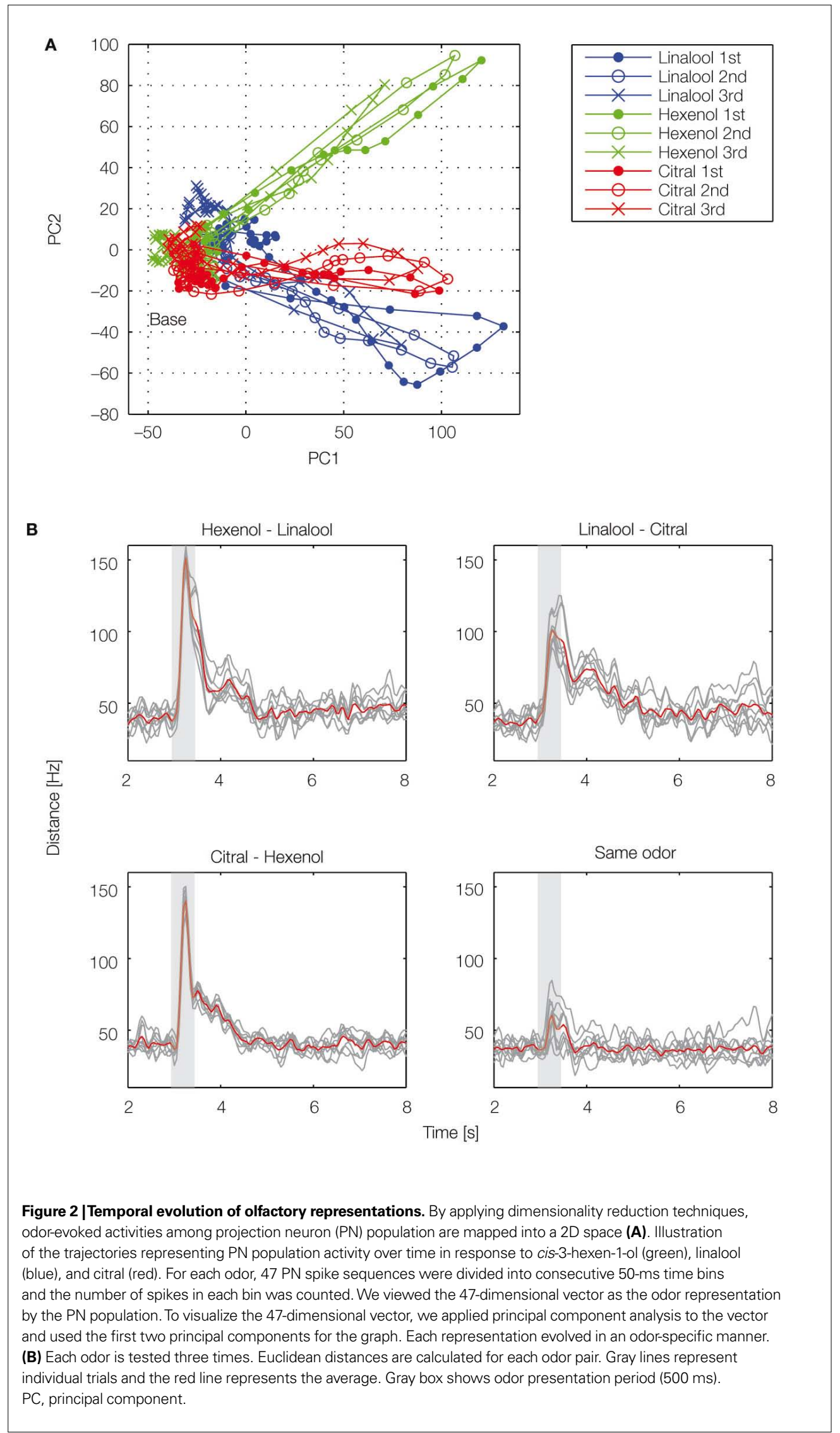



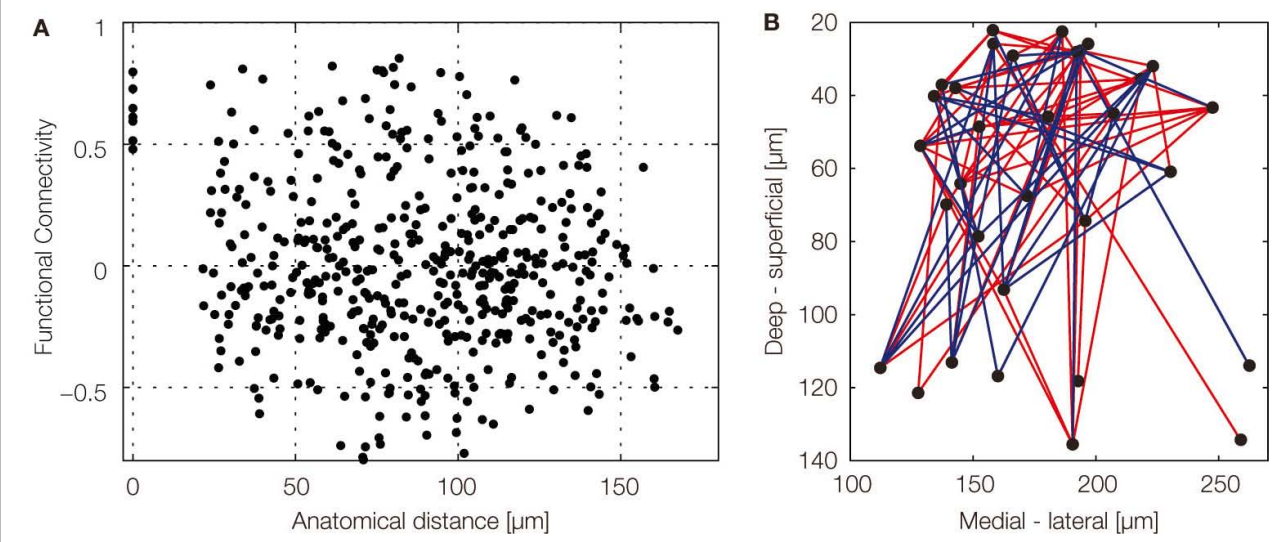

Figure 3 | Spatio-temporal organization of the glomerular network. (A) Functional connectivity as a function of anatomical distance of glomeruli innervated by a pair of projection neurons (PNs). We used Pearson correlation coefficients ( $r$ ) of the responses of pairs of PNs to quantify functional connectivity. Firing rates from stimulus onset to $1 \mathrm{~s}$ after stimulus onset (20 time bins) were used. Pairs of PNs innervating different glomeruli show uncorrelated activity $(r=-0.0556, n=496)$. (B) A graph of functional connectivity mapped onto $x-z$ plane of the antennal lobe. Each node represents a glomerulus. Only strong connectivity is shown, blue and red lines represent positive ( $>0.5)$ and negative (less than -0.5) correlations, respectively. There are many functional connections among glomeruli in the superficial part of the antennal lobe. Although sampling was somewhat sparse, there is a tendency that strong functional connections are rare among deep glomeruli in response to the tested set of plant odors.

could be deduced that different odors elicited distinct dynamics and that the functional connectivity among glomeruli was independent of physical distance (Figure 3), suggesting distance independent lateral inhibition among glomeruli as shown in other species (Olsen et al., 2007; Reisenman et al., 2009). As such, we are able to analyze not only a temporal aspect of sensory representation but also the spatial organization with high resolution. Similar reconstruction approaches incorporating temporal information have been applied in other species as well (Krofczik et al., 2009; Staudacher et al., 2009).

The reconstruction method has several disadvantages. First, the experimental cost is high in comparison with optical imaging methods (Galizia et al., 1999b). Although signal-to-noise ratio of individual recordings is very high, a large number of experiments is required. This could be improved by automation techniques in future (Dunlop et al., 2008). Second, even if we use the same experimental conditions, response variability is present even among the same identifiable neurons in different samples. Individual variability leads to limitations in the temporal resolution at which meaningful information can be extracted. From our results, the firing rate is comparable between different neurons of the same glomerulus at a bin size of approximately 50-100 ms. Faster phenomena such as an odorevoked synchronization among different projec- tion neurons (Laurent, 2002) can apparently only be studied on a per-trial basis using multi-unit recordings.

The variability may partially originate from the internal state of the network (Shu et al., 2003; Stopfer and Laurent, 1999). In such case, variability may be decreased by monitoring physiological parameters of the animal. For example, local field potentials may help for the experimenter to quantify the state of the network. A classification of the responses based on the state of the animal may enable us to perform more precise reconstructions of neural circuits.

\section{TOWARD THE RECONSTRUCTION OF WHOLE INSECT BRAIN}

Our goal lies in between two approaches: From the reconstruction of the sensory map in the antennal lobe and the analysis of its connectivity, we intend to proceed to model circuitry in the brain of the silkmoth that spans from the sensory input to the output of the brain. The ultimate goal will be the emulation of the complete silkmoth brain in software like blue brain project (Markram, 2006). The size of this nervous system is close to the size of a single neocortical column. The model can be evaluated by comparison of behavioral output with real insects by use of insect-robot hybrid systems (Emoto et al., 2007). The key advantages of the silkmoth for this endeavor are access to transgenic and physi- 


\section{Standard brain}

A digital atlas of all structures in the brain. There are several methods to create a standard brain, they generally minimize the individual difference among samples. This is important for estimating synaptic connectivity from neuronal morphology data. ological approaches and a well-defined behavior, pheromone orientation, whose neural substrates have been studied in detail (Kanzaki et al., 2008). This organism is suitable for building a large-scale model due to its good accessibility to the electrophysiology of individual neurons and its nervous system is of the adequate scale to allow simulation of the entire system in real time using currently available supercomputer technology. In addition, neural circuits from sensory to motor neurons via higher order centers in the brain have been investigated in detail by single-cell electrophysiology. The reconstruction approach is well suited to primary sensory neuropils because the stimulus onset provides a clear time reference, but it gradually looses temporal resolution in higher order areas because response variability will increase. However, it is likely that reconstruction techniques as outlined above can be applied to motor systems in which motor output can serve as time reference. Motor areas of the silkmoth have been intensively investigated (Mishima and Kanzaki, 1999; Wada and Kanzaki, 2005) and now we are collecting single-cell recording data from the neurons connecting sensory and motor areas.

For the precise reconstruction of the brain, determining synaptic connectivity is a key problem. Due to the tangled structure of neurons and the huge number of contacts they make, this point has been a long-standing difficulty in any modeling of neural circuits. We employ the insect nervous system in which individual neurons are identifiable and comparable from one individual to another. It therefore becomes possible to integrate data from different experiments in a cumulative way with less conceptual problems. Capitalizing on this property, we will apply multiple techniques to probe synaptic connections including dual electrophysiological recording, optical synaptic probing and transsynaptic tracers. Complementary to this, connectivity for single-neuron data can be estimated through our mapped data base approach merging data in standard brain coordinates. We map the neurons into a standard brain (Brandt et al., 2005; Kurylas et al., 2008) to use this unified structure as a basis to infer potential synaptic contacts (Jacobs and Theunissen, 2000; Stepanyants and Chklovskii, 2005; Tanaka et al., 2004). This will allow the use of realistic multi-compartment biophysical modeling of a large number of neurons in the order of the actual number of neurons in the silkmoth brain.

\section{ACKNOWLEDGMENTS}

This research was supported by a grant from a Grant-in-Aid for Scientific Research (B) from the Ministry of Education, Culture, Sports, Science and Technology.

\section{REFERENCES}

Bacon, J. P., and Murphey, R. K. (1984). Receptive fields of cricket giant interneurons are related to their dendritic structure. J. Physiol. 352, 601-623.

Bjaalie, J.G., Leergaard, T. B., Lillehaug, S., Odeh, F., Moene, I.A., Kjode, J. O., and Darin, D. (2005). Database and tools for analysis of topographic organization and map transformations in major projection systems of the brain. Neuroscience 136, 681-695.

Brandt, R., Rohlfing, T., Rybak, J., Krofczik, S., Maye, A., Westerhoff, M., Hege, H. C., and Menzel, R. (2005). Three-dimensional average-shape atlas of the honeybee brain and its applications. J. Comp. Neurol. 492, 1-19.

Cassenaer, S., and Laurent, G. (2007). Hebbian STDP in mushroom bodies facilitates the synchronous flow of olfactory information in locusts. Nature 448, 709-713.

Comer,C.M., and Robertson, R.M.(2001). Identified nerve cells and insect behavior. Prog. Neurobiol. 63, 409-439.

Delcomyn, F. (2004). Insect walking and robotics. Annu. Rev. Entomol. 49, 51-70.
Dunlop, J., Bowlby,M.,Peri, R., Vasilyev, D., and Arias, R. (2008). High-throughput electrophysiology: an emerging paradigm for ion-channel screening and physiology. Nat. Rev. Drug Discov. 7, 358-368.

Eaton, R.C.,Lee,R.K.K., and Foreman, M. B. (2001). The Mauthner cell and other identified neurons of the brainstem escape network of fish. Prog. Neurobiol. 63, 467-485.

Emoto, S., Ando, N., Takahashi, H., and Kanzaki, R. (2007). Insect-controlled robot - evaluation of adaptation ability. J. Robot. Mechatron. 19, 436-443.

Fukushima, R., and Kanzaki, R. (2009). Modular subdivision of mushroom bodies by Kenyon cells in the silkmoth. J. Comp. Neurol. 513, 315-330.

Galizia, C. G., Mcllwrath, S. L., and Menzel, R. (1999a). A digital-three dimensional atlas of the honeybee antennal lobe based on optical sections acquired by confocal microscopy. Cell Tissue Res. 295, 383-394.

Galizia, C. G., Sachse, S., Rappert, A., and Menzel, R. (1999b). The glomerular code for odor representation is species specific in the honeybee Apis mellifera. Nat. Neurosci. 2, 473-478.
Hallem, E. A., and Carlson, J. R. (2006). Coding of odors by a receptor repertoire. Cell 125, 143-160.

Hawkins, R. D.,Abrams, T.W., Carew, T. J., and Kandel, E. R. (1983). A cellular mechanism of classical conditioning in Aplysia: activity-dependent amplification of presynaptic facilitation. Science 219, 400-405.

Heinze, S., and Homberg, U. (2007) Maplike representation of celestial E-vector orientations in the brain of an insect. Science 315, 995-997.

Hildebrand, J. G., and Shepherd, G. M. (1997). Mechanisms of olfactory discrimination: converging evidence for common principle across phyla. Annu. Rev. Neurosci. 20, 595-631.

Hodgkin, A. L., and Huxley, A. F. (1952). A quantitative description of membrane current and its application to conduction and excitation in nerve. J. Physiol. 117, 500-544.

Jacobs, G.A., and Theunissen, F. E. (1996) Functional organization of a neural map in the cricket cercal sensory system. J. Neurosci. 16, 769-784.

Jacobs, G. A., and Theunissen, F. E. (2000) Extraction of sensory parameters from a neuralmapbyprimarysensoryinterneurons. J. Neurosci. 20, 2934-2943.
Jefferis, G. S., Potter, C. J., Chan, A. M., Marin, E. C., Rohlfing, T., Maurer, C. R. Jr., and Luo, L. (2007). Comprehensive maps of Drosophila higher olfactory centers: spatially segregated fruit and pheromone representation. Cell 128, 1187-1203.

Kanzaki, R., Ando, N., Sakurai, T., and Kazawa, T. (2008). Understanding and reconstruction of the mobiligence of insects employing multiscale biological approaches and robotics. $A d v$. Robot. 22, 1605-1628.

Kazawa, T., Ikeno, H., and Kanzaki, R. (2008). Development and application of a neuroinformatics environment for neuroscience and neuroethology. Neural Netw. 21, 1047-1055.

Kazawa, T., Namiki, S., Fukushima, R., Terada, M., Soo, K., and Kanzaki, R. (2009). Constancy and variability of glomerular organization in the antennal lobe of the silkmoth. Cell Tissue Res. 336, 119-136.

Krofczik, S., Menzel, R., and Nawrot, M. P. (2009). Rapid odor processing in the honeybee antennal lobe network. Front. Comput. Neurosci. 2, 9.

Kurylas, A. E., Rohlfing, T., Krofczik, S., Jenett, A., and Homberg, U. (2008). 
Standardized atlas of the brain of the desert locust, Schistocerca gregaria. Cell Tissue Res. 333, 125-145.

Laurent, G. (2002). Olfactory network dynamics and the coding of multidimensional signals. Nat. Rev. Neurosci. 3, 884-895.

Lindemann, J.P., Weiss, H., Möller, R., and Egelhaaf, M. (2008). Saccadic flight strategy facilitates collision avoidance: closed-loop performance of a cyberfly. Biol. Cybern. 98, 213-227.

Marder, E., and Bucher, D. (2007). Understanding circuit dynamics using the stomatogastric nervous system of lobsters and crabs. Annu. Rev. Physiol. 69, 291-316.

Markram,H. (2006). Theblue brain project. Nat. Rev. Neurosci. 7, 153-160.

Mishima, T., and Kanzaki, R. (1999). Physiological and morphological characterization of olfactory descending interneurons of the male silkworm moth, Bombyx mori. J. Comp. Physiol. A 184, 143-160.

Namiki, S., and Kanzaki, R. (2008). Reconstructing the population activity of olfactory output neurons that innervate identifiable processing units. Front. Neural Circuits 2, 1

Olsen, S. R., Bhandawat, V., and Wilson, R. I. (2007). Excitatory interactions between olfactory processing channels in the Drosophila antennal lobe. Neuron 54, 89-103.
Reichardt, W. (1957). AutokorrelationsAuswertung als Funktionsprinzip des Zentralnervensystems (bei der optischen Wahrnehmung eines Insektes). $Z$. Naturforsch. B 12, 448-457.

Reisenman, C. E., Chirstensen, T. A., and Hildebrand, J. G. (2005). Chemosensory selectivity of output neurons innervating an identified, sexually isomorphic olfactory glomerulus. J. Neurosci. 25, 8017-8026.

Reisenman, C. E., Heinbockel, T., and Hildebrand J. G. (2009). Inhibitory interactions among olfactory glomeruli do not necessarily reflect spatial proximity. J. Neurophysiol. 100, 554-564.

Ritzmann, R. E., and Büschges, A. (2007). Adaptive motor behavior in insects. Curr. Opin. Neurobiol. 17, 629-636.

Rospars, J. P. (1983). Invariance and sexspecific variations of the glomerular organization in the antennal lobes of a moth, Mamestra brassicae, and a butterfly, Pieris brassicae.J. Comp. Neurol. 220, 80-96.

Seki, Y., and Kanzaki, R. (2008) Comprehensive morphological identification and GABA immunoreactivity of antennal lobe local interneurons in Bombyx mori.J. Comp. Neurol. 506, 93-107.

Shu, Y., Hasenstaub, A., and McCormick, D. A. (2003). Turning on and off recurrent balanced cortical activity. Nature 423:288-293.
Staudacher, E. M., Huetteroth, W., Schachtner, J., and Daly, K. C. (2009). A 4-dimensional representation of antennal lobe output based on an ensemble of characterized projection neurons. J. Neurosci. Methods 180, 208-223.

Stepanyants, A., and Chklovskii, D. B. (2005). Neurogeometry and potential synaptic connectivity. Trends Neurosci. $28,387-394$.

Stopfer, M., and Laurent, G. (1999). Shortterm memory in olfactory network dynamics. Nature 402, 664-668.

Tanaka, N.K.,Awasaki, T., Shimada, T., and Ito, K. (2004). Integration of chemosensory pathways in the Drosophila second-order olfactory centers. Curr. Biol. 14, 449-457.

Troyer, T. W., Levin, J. E., and Jacobs, G. A (1994). Construction and analysis of a database representing a neural map. Microsc. Res. Tech. 29, 329-343.

Urata, H., Otsuka, A., Isokawa, T., Seki, Y., Kamiura, N., Matsui, N., Ikeno,H., and Kanzaki, R. (2006). On self-organizing map based classification of insect neurons. In Proceedings of the IEEE Region 10 Conference, TENCON 2006, IP 5.2, TEN-561. Hong Kong, IEEE

Vosshall, L. B., and Stocker, R. F. (2007) Molecular architecture of smell and taste in Drosophila. Annu. Rev. Neurosci. 30, 505-533.

Wada, S., and Kanzaki, R. (2005). Neural control mechanisms of the pheromone- triggered programmed behavior in male silkmoths revealed by double-labeling of descending interneurons and a motor neuron. J. Comp. Neurol. 484, 168-182.

Webb, B. (2002). Robots in invertebrate neuroscience. Nature 417, 359-363.

White, J.G., Southgate, E., Thomson, J.N., and Brenner, S. (1986). The structure of the nervous system of the nematode Caenorhabditis elegans. Philos. Trans. R. Soc. Lond., B, Biol. Sci. 314, 1-340.

Wilson, R.I., Turner, G. C., and Laurent, G. (2004). Transformation of olfactory representations in the Drosophila antennal lobe. Science 303, 366-370.

Conflict of InterestStatement:The authors declare that the research was conducted in the absence of any commercial or financial relationships that could be construed as a potential conflict of interest.

Received: 02 May 2009; paper pending published: 28 June 2009; accepted: 13 July 2009; published: 15 September 2009. Citation: Front. Neurosci. (2009) 3, 2: 206 213. doi: 10.3389/neuro.01.028.2009 Copyright $\odot 2009$ Namiki, Haupt, Kazawa, Takashima, Ikeno and Kanzaki. This is an open-access article subject to an exclusive license agreement between the authors and the Frontiers Research Foundation, which permits unrestricted use, distribution, and reproduction in any medium, provided the original authors and source are credited. 